\title{
Design research based on deconstruction and reorganization of design methods of children's tableware
}

\author{
Wenming Liu ${ }^{1}$, Wanqi Yan ${ }^{1 *}$ \\ ${ }^{1}$ Shenyang Jianzhu University, Shenyang, Liaoning, 110168, China
}

\begin{abstract}
This study is aimed at children aged 5-7 years old. Though observations, investigations and analysis of children's psychological characteristics, preferences and interests, tableware designs for children in preschool and primary school are studied. Design methods of deconstruction and recombination are applied to better integrate Chinese mythological and cartoon elements that children are interested in daily life with the designs, which plays a good role in designs of children's tableware. Methods of deconstruction and recombination in product designs are summarized, which can also provide some guidance for future product designs.
\end{abstract}

\section{Design statuses of deconstruction and reorganization}

With developments of society, the growth of children is an important concern of families, so, only design works that can reflect the needs of users to a greater extent can be favoured by the public.

\subsection{Development trend}

Because people are living in different times, they have different ways of thinking, living habits and family factors. Children with an age of 5-7 are in a period of physical and mental development [1], when their desire for knowledge is at its strongest level, therefore, demands for children's products are also changing constantly. On the one hand, children's products should meet their living needs; on the other hand, the cultural characteristics of new products should be used to exert a subtle influence on children's cognition. However, deconstruction and reorganization give products different usage meanings at the same time, which will bring different interactive experiences to children. Therefore, deconstruction and reorganization are gradually widely used in product designs. At present, applications of deconstruction and reorganization in products are mainly extractions, simplifications and reorganizations of various types of elements.

\subsection{The brief of deconstruction and reorganization}

Deconstruction is a very useful design method in product designs. According to needs of designs, essence and dregs of deconstructed objects are selected and discarded and then added to the reserve list of design symbol elements and reconstructed in new designs to form a new product image. In designs, such artistic symbols can help designer's to design works so as to obtain the identity of information, reflect personalities and further obtain the power of styles and cultures, which is one of the important design methods in art designs. As long as the method of deconstructing element decomposition can be used naturally without breaking individual characters, valuable products can be created. In addition, after restructuring deconstructs element combinations according to the balance among different rules, germinations can be a variety of different creative products and new products can be elements of original host cultural connotations of a kind of inheritance, while in fact, it is another new unique original product.

\section{Products of deconstruction and reorganization}

Using methods of deconstruction and reorganization in processes of product designs can make main ideas of designers to be shown in new products and provide a steady stream of inspirations for them. Designers can be blended in these deconstructions of abstract symbols in products, so as to bring new product use values.

\subsection{Main elements of deconstruction and reorganization}

Products that can be used to basically deconstruct and reorganize has the following several factors: cultural, popular, text and graphic elements, etc. So early in product designs, designers need to find user demands through a wide range of researches in selecting and matching the design elements.

\footnotetext{
*Corresponding author's e-mail: 980383752@qq.com
} 


\subsection{1. the cultural element.}

Products, as carriers of cultures, are in an era with generations of words, contemporary cultures as well as designs of the products, which provide cultural spirits and emotional experiences of design elements. Cultural element are on the expansion of existing creative cultural connotations, and it is also designers of abstract cultures that put them into designs of products. Cultural elements in modeling elements will contain cultural symbol design elements and color collocations, etc.

Cultural modeling elements originate from natural lives, some of which are also from local humanities. New cultural elements used in product designs can impact on cultural heritages, for new products spread humanity. Collocations of cultural elements to add color element modeling will give vitality and cultural elements. Different colors can produce different visual feelings, and due to factors such as gender, age and geography, etc., people will have different color preferences. As is shown in the Figure 1, the author takes traditional culture of "zodiac" life as the theme, each zodiac animal head is respectively formed to carry on analyses and designs, and then colors are matched according to properties of different animals, thus constituting a set of shape designs of novel materials.

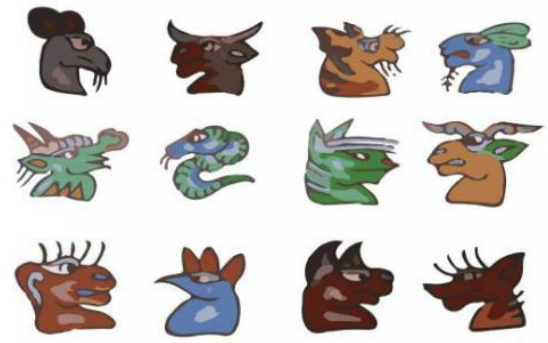

Figure 1. The Chinese zodiac elements.

\subsubsection{The popular element.}

Along with progresses of society, people's ideas and ways of life are also rising and changing. So, product designs must be emphasized on seizing the favorite and popular elements of current target crowds. This can not only greatly attract consumers, but also design new products timely. There are all kinds of popular elements, such as films and televisions, literature, music, clothing and fun projects, etc.

As for children at this age, they choose popular elements in product designs, they have strong interests in humor cartoon elements, which are closer to life of children, and are easily accepted by them. So, adding animated cartoon elements in designs of children products is a design way of thinking that is worth considering.

\subsubsection{The text element.}

The invention of texts to improve communication difficulties makes the fleeting mandarin spread in different places. Every country has its own languages. In design, it is usually the same meaning, texts with similar shapes adopt deconstruction and reorganization to process into new text decoration elements. Explanatory text elements referring to stand out can often express the most accurate semantics to consumers. Designers can use text elements in product designs for decorations, so as to visually express their main design purposes.

\subsubsection{The graphic elements.}

A graphic is a depiction of objective things that are identical and realistic. It is commonly used in human social activities and information dissemination media, which is an important element in visual interactions.

\subsection{Products of deconstruction and reorganization}

For users, a high-quality diversified product not only can meet their diverse needs, but also greatly improve their living standards, while for designers, products are a bridge to communicate with users. Deconstructions and reorganizations in products are not only recombination and fusions of basic elements, but also innovative developments of product functions. Only by constantly adding new elements can products become more dynamic.

Since the design theme of each product is different, different elements are needed to match with them. For example, among a set of bookmarks of imperial seals in cultural creation products of the Imperial Palace, the designer selected six of them from the seals of three emperors (Kangxi, Yongzheng and Qianlong) and made a set of exquisite hollow copper bookmarks. In the overall form, this set of bookmarks are mainly about inscriptions of emperor seals, including text, cultural and other design elements[2].

\section{The application of deconstruction and reorganization in product design method researches}

\subsection{The Design principles}

\subsubsection{Uniqueness and innovation principles.}

For products, only with uniqueness and innovations can they highlight their characteristics and attract consumers' desires to purchase. Designers should design according to users through selecting main elements in different categories, while this does not mean that a simple element symbol should be directly extracted and applied to product designs, instead, it should be appropriate deconstructions and reorganizations of multiple elements among them, and in this way we can make unique product innovations. It becomes a trend in the present society. For example, children's products can be designed with combinations of cartoon and text elements, while adult products should be emphasized on integration and innovations of various rational elements in designs. By 
making products unique and innovative, freshness of users can be kept through using the products.

\subsubsection{The Matching principle.}

Practicality is a basic function that every product must have. In product designs, designers need to analyze and summarize users on a large scale, and finally select elements accurately matched with users according to research results. If a product cannot meet the needs of users in terms of practicality[3], there will be no consumer market for such products. Therefore, it is necessary to pay attention to the combination of products and practicality in product designs, which are peopleoriented designs in order to create a high-quality life for users.

\subsubsection{The principle of diversification.}

Diversification can reveal products more vitality. Designers need to grasp a variety of elements when designing products. User's demands of products are always diversified, so product designs should be based on user's needs, and designers should pay attention to collocations of various elements.

\subsection{The design methods of deconstruction and reorganization of researches}

\subsubsection{The combination of abstract and concrete.}

In product designs, most of collected elements cannot be directly used. Some elements are abstract descriptions and ancient writing across the ages, and there is even a synonym of trends of The Times. Therefore, designers need to combine abstract elements with concrete elements in real life and integrate them into designs through deconstructions of elements according to their design purposes, which can make product design processes easier. The combination of abstract and concrete is like a process of translating complex classical Chinese into plain Chinese. By combining a large number of single abstract elements with tangible things in life, a redesign is taken after recombination and innovations, which can make new products more emotional and interesting. Then functions of the products will be endowed with souls, which are even more acceptable. Only through new elements can products become more dynamic.

For example, the Page Piggy's cultural creative products are the most popular among children. The Page is an abstract element of cartoon that is reconstructed and innovated with the figurative image of a pig. The last, the designer combines it with products that make it into a widely accepted derivative[4].

\subsubsection{Methods of element matching and recombination.}

Designing elements needs to be matched with selections based on user locations, which is like the fact that the young should wear children's clothes while the older should wear adult clothes. There are many different styles of clothing matched between them, so designing elements also need to match with each other, and they should be of different styles. Such as the fact that it needs to choose adults' favorite elements when working in adult product designing. Product that adults think mature tend to have rationality and be contracted, so when choosing elements, contracted elements of Nordic styles as well as character and graph elements can be chosen. Finally, it is used in combination, which reflects application of deconstructions and recombination of elements in designs[5].

For example, plaid pattern and text elements are used on BURBERRY's plaid tote bags, which recombine basic tote bags in the design, giving people a kind of simple and fashionable beauty.

\subsection{Design practice and case presentation.}

\subsubsection{Analysis of customer needs and positioning.}

Children between 5 and 7 years old have the most abundant imagination and creativity. At this stage, children's desires for unknown things are also very strong, so things in children's life can often be contacted, which should play a role of guiding and stimulating their learning desires. Besides, it is necessary to get a novel and creative design in children's life. Such a design can bring children an infinite imagination space and let them grow up happily.

Through market researches, children's tableware first need to conform to their living habits and psychological characteristics of loving the new and loathing the old; secondly, they need to be affordable for parents; for designers or producers, it is necessary for children's meals to have characteristics of low-cost diversifications.

\subsubsection{The project design.}

On the basis of crowd location, select elements that are easier for children to accept. Finally, cultural and popular elements are selected and combined in designs of children's tableware. For children, such products need to meet not only their most basic practical needs, but also some special psychological demands of them.

As shown in the Table 1, elements that children are interested in and are easy for them to accept are listed respectively in the table. Then, these elements are categorized according to the categorization of designing elements. And the preference scale (1 to 9) is used, which the highest value indicates the highest degree of preference. The cartoon and story are 9; the astronomy is 8 and kids doodle is 6 . The elements are listed in descending order of children's preference. The most popular element for children is cartoon element followed 
by story element. In designing, cartoon falls into popularity element category, with both story and astronomic knowledge falling into culture element category and children's graffiti falling into graph element category.

Table 1. The children's favorite elements.

\begin{tabular}{|c|c|c|c|c|}
\hline & Cartoon & Story & Astronomy & Kids doodle \\
\hline Types of elements & $\begin{array}{c}\text { The popular } \\
\text { element }\end{array}$ & $\begin{array}{c}\text { The cultural } \\
\text { element }\end{array}$ & The cultural element & $\begin{array}{c}\text { The graphic } \\
\text { elements }\end{array}$ \\
\hline $\begin{array}{c}\text { Degree of preference } \\
(1-9)\end{array}$ & 9 & 9 & 7 & 6 \\
\hline
\end{tabular}

It is determined that cultural elements are mainly the four gods and beasts in Chinese culture, and those popular elements are mainly in cartoons. The four sacred animals, known as the four images in traditional Chinese culture, are products of ancient mythologies and astronomy. The five elements and directions of philosophy have been integrated into Chinese culture, which have different directions and colors: cyan for wood in the east, white for gold in the west, red for fire in the south, and ink for water level in the north. Therefore, according to design principles of deconstruction and recombination in product designs, color tones of cartoon image elements are mainly composed of a blue dragon, a white tiger, a rosefinch and a Xuanwu, which were respectively determined to be cyan, white, vermilion and dark green.

Therefore, the color keynote of cartoon image elements based on mythical creatures such as the Dragon, the White Tiger, the Suzaku and the Basalt were determined to be a cyan, white, scarlet, and dark green by deconstructing and reorganizing design principles in product design. The abstraction and concretization combination method of the deconstruction and reorganization method in product design was utilized to integrate the 4 mythical creatures such as the Kylin, the
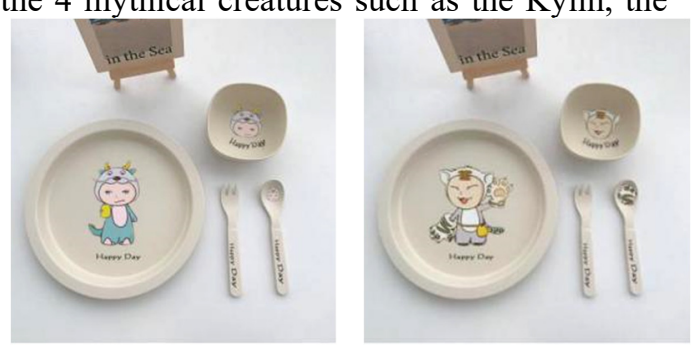

Phoenix, the Turtle and the Dragon described in the Chapter IV of "the Book of Rites·The Ninth" with the tiger, the turtle, the bird and the snake in real life to design images with repetitive modification. And then according to the element matching and reorganization method of the deconstruction and reorganization method in product design the final design of the cartoon image elements of the four mythical creatures show in Figure 2 was completed by combining images of the four creatures with animation images of popular elements for children. In addition, letters and tableware were integrated into the design of children's tableware after their reorganization. The design elements on spoons and bowls are from a detail pattern after element innovation. In this way, the children's tableware innovatively designed through the deconstruction and reorganization method is characterized by diversity and cultural significance, which not only meet practical demand of children and parents, but also empower inheritance of Chinese culture. In addition, they enable children at a stage of constant exploration of new things to understand and learn culture from life, and this subtle influence can also bring greater consumer markets and profits to this product.
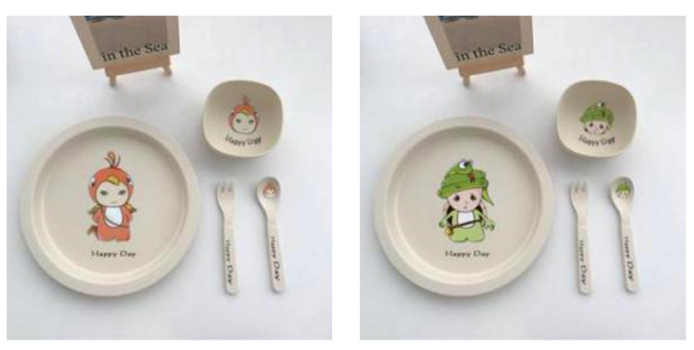

Figure 2. The four mythical creatures children tableware.

\section{Conclusion}

It can be seen that the application rate of deconstruction and reorganization in product design is very high, which complies with design principles such as matching, diversity, uniqueness and innovation. The design method of deconstruction and reorganization in product design can be used to design new products that fully demonstrate their personalized and diverse characteristics, and products like this are easy to be accepted and loved by the public.

\section{References}

1. Xiangyu Kuang. (2007) The Characteristics of products for children aged 4-5 years. [J]. Neijiang technology, (01): 109-111.

2. Zhengbo Qu, Hongda Du, Wenqiang Ji. (2017) The brief analysis of deconstruction in modern product design. [J]. Industrial design, (01): 121-122.

3. Jia Liang, Qingzheng Xiong. (2014) Research on creative demand design of children's product development. [J]. Packaging engineering, (18): 6870 . 
4. Jiaqi Zhang, Yulu Dai. (2018) Research and application of cultural derivative design based on product semantics. [J]. Art panorama, (11): 96-97.

5. Ping Lu. (2013) On the artistic expression of deconstruction technique in modern clothing design. [J]. Teaching of forestry region, (12): 33-34. 